NWU-11/95

Sept. 1995

\title{
Classical and Quantum Solutions and the Problem of Time in $R^{2}$ Cosmology
}

\author{
Masakatsu Kenmoku 1 and Kaori Otsuki 円 \\ Department of Physics \\ Nara Women's University, Nara 630, Japan \\ Kazuyasu Shigemoto [5 and Kunihiko Uehara \\ Department of Physics \\ Tezukayama University, Nara 631, Japan
}

\begin{abstract}
We have studied various classical solutions in $R^{2}$ cosmology. Especially we have obtained general classical solutions in pure $R^{2}$ cosmology. Even in the quantum theory, we can solve the Wheeler-DeWitt equation in pure $R^{2}$ cosmology exactly. Comparing these classical and quantum solutions in $R^{2}$ cosmology, we have studied the problem of time in general relativity.
\end{abstract}

\footnotetext{
${ }^{1}$ E-mail address:kenmoku@nara-wu.ac.jp

${ }^{2}$ E-mail address: sp19254@nara-wu.ac.jp

${ }^{3}$ E-mail address:shigemot@tezukayama-u.ac.jp

${ }^{4}$ E-mail address:uehara@tezukayama-u.ac.jp
} 


\section{Introduction}

Recently quantum theoretical treatment of gravity has been paid attention in various topics such as the time development of quite early universe [1, 2], exact treatment of Hawking radiation [3] and the problem of time in cosmology [4]-[7].

On the other hand, attempts to generalize Einstein's gravitation theory have a long history in various purposes. The generalized Einstein theory with additional $R^{2}$ term was introduced to regularize ultraviolet divergences in the Einstein theory [8]. It was applied to cosmology to obtain the bounce universe to avoid the singularity at the creation of the universe[9]. The structure and the properties of the higher derivative gravitation theory were further elaborated in subsequent works 10.

Other people have tried to generalize Einstein theory to modify Newtonian force laws from the framework of general relativity [11, 12]. Since there is no established direct observation of dark matter, there are many attempts to explain rotation curves without dark matter by modifying the Newtonian force empirically[13] or from the framework of the general relativity[14]. Whereas, on the application of the generalized Einstein theory to cosmology, many people are interested in $R^{2}$ cosmology as it may give a promising model to explain the inflation in early universe without using the fictitious scalar field [15, 16]. The semiclassical solution of higher-order cosmology gives the de Sitter universe [17, but there arises instability in the quantum treatment 18 .

In the previous paper, we attempted to explain not only the flat rotation curves of spiral galaxies but also the large-scale structure of the universe, starting from a simple model with adding $R^{2}$ term to the Einstein action [14]. For the generalized Einstein action, we have the quite stringent observational constraint that the coefficient $\gamma$ of the Robertson expansion[19] must be quite near 1 from the classical tests of general relativity by the radar echo delay experiment. However, in the previous paper, we constructed the model which gives $\gamma=1$ at solar scale and gives Sander's type gravitational potential at galaxy scale 20.

In series of our papers, we applied our $R^{2}$ involved gravitational theory to 1) solar scale, 2) galaxy scale, 3) large-scale of the universe, such as "Great Wall", in classical level. Then in this paper, we will apply this $R^{2}$ involved gravitational theory to cosmology. First we will find various classical solutions in $R^{2}$ cosmology. Especially we will give general classical solutions in pure $R^{2}$ cosmology. Next we will consider the quantum theory of pure $R^{2}$ cosmology, and will give exact solutions of Wheeler-DeWitt equation. Comparing these classical and quantum solutions in $R^{2}$ cosmology, we will study the problem of time in the general relativity according to the de Broglie-Bohm interpretation[21]. 


\section{Classical Solutions of $R^{2}$ Cosmology}

We use Weinberg notation [19] and consider the following generalized action:

$$
I \quad=\int d^{4} x \sqrt{-g}\left[-\frac{1}{16 \pi G}\left(R+\alpha R^{2}\right)+\mathcal{L}_{\text {matter }}\right]
$$

where $G$ is the gravitational constant, $R$ is the scalar curvature and $\alpha$ is a constant.

In order to study classical and quantum solutions in $R^{2}$ cosmology, we consider the homogeneous and the isotropic space-time with the metric

$$
d s^{2}=-b(t)^{2} d t^{2}+a(t)^{2}\left[d r^{2}+r^{2}\left(d \theta^{2}+\sin \theta^{2} d \varphi^{2}\right)\right],
$$

where we put the curvature term to be zero.

Using this metric, we obtain the expression of the scalar curvature

$$
R=-6\left(\frac{\ddot{a}}{a b^{2}}+\frac{\dot{a}^{2}}{a^{2} b^{2}}-\frac{\dot{a} \dot{b}}{a b^{3}}\right)
$$

After partial integration, the original action can be transformed into the form

$$
I=\int d^{3} x \int d t\left[-\frac{3}{8 \pi G}\left\{\frac{a \dot{a}^{2}}{b}+6 \alpha\left(\frac{\ddot{a}^{2} a}{b^{3}}-\frac{2 \ddot{a} \dot{a} \dot{b} a}{b^{4}}+\frac{\dot{a}^{2} \dot{b}^{2} a}{b^{5}}+\frac{\dot{a}^{4}}{a b^{3}}\right)\right\}+a^{3} b \mathcal{L}_{\text {matter }}\right]
$$

For perfect fluid approximation of matter, equations of motion to be solved become

$$
\begin{aligned}
& H^{2}-6 \alpha\left\{2 \ddot{H} H-\dot{H}^{2}+6 \dot{H} H^{2}\right\}=\frac{8 \pi G}{3} \rho, \\
& \dot{\rho}+3 H(\rho+P)=0,
\end{aligned}
$$

where we put $b=1$ after variation. We use notation $H=\dot{a} / a$, and denote $\rho$ and $P$ as density and pressure of perfect fluid respectively. 


\subsection{Classical solution with perfect fluid in pure $R^{2}$ cosmology}

We put $P=\gamma \rho$ in this case, then equations to be solved become in the form:

$$
\begin{aligned}
& -6 \alpha\left\{2 \ddot{H} H-\dot{H}^{2}+6 \dot{H} H^{2}\right\}=\frac{8 \pi G}{3} \rho, \\
& \dot{\rho}+3 H(1+\gamma) \rho=0 .
\end{aligned}
$$

From Eq.(8), we obtain $\rho a^{3(1+\gamma)}=\rho_{0} a_{0}{ }^{3(1+\gamma)}=$ (const.). Substituting this relation into Eq.(7), we obtain the following power type solution of the universe:

$$
\begin{aligned}
a(t) & =k_{1} t^{\frac{4}{3(1+\gamma)}}, \\
\text { with } \quad k_{1} & =a_{0}\left(\frac{\pi G \rho_{0}(1+\gamma)^{3}}{4 \alpha(5-3 \gamma)}\right)^{\frac{1}{3(1+\gamma)}},
\end{aligned}
$$

where we use the initial condition $a(0)=0$.

\subsection{Classical solution without matter in $R+\alpha R^{2}$ cosmology}

a) Case of $\alpha<0$

In this case, equation to be solved becomes in the form[15, 16]:

$$
H^{2}-6 \alpha\left\{2 \ddot{H} H-\dot{H}^{2}+6 \dot{H} H^{2}\right\}=0 .
$$

When we denote $\tilde{\alpha}=-\alpha(>0)$ and change variables into forms

$$
\begin{aligned}
H(t) & =\frac{\sqrt{6}}{36 \sqrt{\tilde{\alpha}}} f(\xi)^{2}, \\
t & =\sqrt{24 \tilde{\alpha}} \xi,
\end{aligned}
$$

we obtain following equation: 


$$
\frac{d^{2} f}{d \xi^{2}}+f^{2} \frac{d f}{d \xi}+f=0
$$

except the uninteresting trivial case $f=0$ (static universe).

Though we cannot find exact solution for Eq.(12), we can solve that equation perturbatively. First, treating the third term in Eq.(12) as the perturbation, we obtain the following power series solution:

$$
f(\xi)=\sqrt{\frac{3}{2 \xi}}\left[1-\frac{2}{3} \xi^{2}-\frac{2}{25} \xi^{4}+\frac{4}{4725} \xi^{6}+\frac{23186}{4606878} \xi^{8}+\frac{6428}{4558125} \xi^{10}+\cdots\right]
$$

where we assumed that singularity occures at $\xi=0$.

Second, treating the second term in Eq.(12) as the perturbation, we obtain the following oscillating solution by taking the initial condition $f(0)=0$ :

$$
f(\xi)=\sin \xi+\left[\frac{1}{32}(\cos \xi-\cos 3 \xi)-\frac{\xi}{8} \sin \xi\right]+\cdots .
$$

Combining these perturbative solutions, we know the asymptotic behavior $f(\xi) \sim \sin (\xi-$ $\left.\xi_{0}\right) / \sqrt{\xi}$ as $\xi \rightarrow \infty$.

Here we must notice that the above solution does not approach the solution of equation $H^{2}=0$, which comes from Eq.(10) by putting $\tilde{\alpha}=0$ from the beginning, even in the limit $\tilde{\alpha} \rightarrow 0$. This is because $\tilde{\alpha}$ is the coefficient of the highest derivative term. Then the limit $\tilde{\alpha} \rightarrow 0$ give the quite chaotic behavior, which is quite similar to the turbulent limit in the Navier-Stokes equation. Actually, $H(t)$ has the following $t$ dependence:

$$
H(t)=\frac{\sqrt{6}}{36 \sqrt{\tilde{\alpha}}} f^{2}\left(\frac{t}{\sqrt{24 \tilde{\alpha}}}\right) .
$$

In the limit $\tilde{\alpha} \rightarrow 0$ with finite $t, H(t)$ oscillates quite rapidly and the oscillation amplitude diverges, and finally becomes chaotic.

b) Case of $\alpha>0$ 
We define $\tilde{\alpha}=\alpha(>0)$ in this case and use the same notation as those of Eq.(12), then we have the following equation:

$$
\frac{d^{2} f}{d \xi^{2}}+f^{2} \frac{d f}{d \xi}-f=0 .
$$

We cannot solve exactly in this case either, but we can see that $f(\xi) \sim \sqrt{2\left(\xi-\xi_{0}\right)}$ as $\xi \rightarrow \infty$.

\subsection{Classical solution without matter in pure $R^{2}$}

In this case we put $H(t)=f^{2}(t)$, then equation of motion to be solved becomes in the form $\frac{d^{2} f}{d t^{2}}+\frac{d f^{3}}{d t}=0$, in addition to the trivial case $f=0$. The first integral of this equation gives $\frac{d f}{d t}+f^{3}=-c_{1}^{3}$, where $c_{1}$ is the integration constant. The special solution of this equation is given by $f=-c_{1}=$ (const.), which gives the de Sitter universe $a(t)=a(0) \exp c_{1}^{2} t$. Most general solution is given in the following implicit form:

$$
\frac{1}{6 c_{1}^{2}} \log \left(\frac{\left(f+c_{1}\right)^{3}}{f^{3}+c_{1}^{3}}\right)+\frac{1}{\sqrt{3} c_{1}^{2}} \arctan \frac{2 f-c_{1}}{\sqrt{3} c_{1}}=\left(t_{0}-t\right) .
$$

In $c_{1}=0$ case, we can express $f$ in the explicit form $f=1 / \sqrt{2\left(t-t_{0}\right)}$, which gives the square root type solution of the universe

$$
a(t)=a_{0} \sqrt{t-t_{0}}
$$

\section{Another Formulation of $R^{2}$ Cosmology}

In order to reduce the higher derivative term, we introduce an auxiliary field $\sigma$ and rewrite the original action in the form: 


$$
I=\int d^{4} x \sqrt{-g}\left[-\frac{1}{16 \pi G}\left(R+\alpha\left(-2 R \sigma-\sigma^{2}\right)\right)+\mathcal{L}_{\text {matter }}\right] .
$$

This reproduces the original action after substituting the solution of $\sigma$ field. In the quantum theory, taking the path integral formulation, we have additional term $\prod_{x} 1 /[-g(x)]^{1 / 4}$ when we integrate over $\sigma$. This additional factor changes the measure of the inner product, which is connected to way how to define the ordering of operators. In this sense, our quantum theory of $R^{2}$ gravity and conventional quantum theory of $R^{2}$ is different only in the measure. As there is no a priori criterion to determine the measure, here we take above action as the starting action in quantum gravity.

Using the metric of Eq.(22), we obtain

$$
I=\int d^{3} x \int d t\left[-\frac{1}{16 \pi G}\left\{6 \frac{a \dot{a}^{2}}{b}+\alpha a^{3} b\left(12 \sigma\left(\frac{\ddot{a}}{a b^{2}}+\frac{\dot{a}^{2}}{a^{2} b^{2}}-\frac{\dot{a} \dot{b}}{a b^{3}}\right)-\sigma^{2}\right)\right\}+a^{3} b \mathcal{L}_{\text {matter }}\right] .
$$

In pure $R^{2}$ cosmology, we can analize exactly. Then we will consider only pure $R^{2}$ case hereafter. In pure $R^{2}$ cosmology, the Lagrangian density becomes

$$
\mathcal{L}=\frac{\alpha a^{3} b}{16 \pi G}\left(-12 \sigma\left(\frac{\ddot{a}}{a b^{2}}+\frac{\dot{a}^{2}}{a^{2} b^{2}}-\frac{\dot{a} \dot{b}}{a b^{3}}\right)+\sigma^{2}\right) .
$$

We define $X_{+}=a^{3 / 2} \sigma^{3 / 2}, X_{-}=a^{3 / 2}, B=b \sigma^{1 / 2}$, and after partial integral, we obtain 22

$$
\mathcal{L}=\frac{\alpha}{16 \pi G}\left\{\frac{16}{3 B} \dot{X}_{+} \dot{X}_{-}+B X_{+} X_{-}\right\} .
$$

Using this form of Lagrangian, we obtain equations of motion

$$
\begin{aligned}
& \dot{X}_{+} \dot{X}_{-}=\frac{3 B^{2}}{16} X_{+} X_{-}, \\
& \ddot{X}_{ \pm}=\frac{3 B^{2}}{16} X_{ \pm} .
\end{aligned}
$$

Combining these equations, we can obtain general classical solutions in pure $R^{2}$ cosmology by the following two steps. 
Step 1)

First we use temporal time variable $T$, which is defined by the condition $B(T)=1$, and solve equations of motion with this $T$. The metric at this step gives

$$
d s^{2}=-b(T)^{2} d T^{2}+a(T)^{2}\left[d r^{2}+r^{2} d \theta^{2}+\sin \theta^{2} d \varphi^{2}\right] .
$$

Step 2)

The original time variable $t$ and the temporal time variable $T$ is connected with the condition $d t=b(T) d T$, then the classical solution of the original metric is given by $a(t)=$ $a(T(t))$.

In Step 1, equations we deal with are followings:

$$
\begin{aligned}
& \frac{d X_{+}}{d T} \frac{d X_{-}}{d T}=\frac{3}{16} X_{+} X_{-}, \\
& \frac{d^{2} X_{ \pm}}{d T^{2}}=\frac{3}{16} X_{ \pm},
\end{aligned}
$$

which come from Eq.(23) after substituting $B=1$. Then we get general solutions of the following three types:

$$
\begin{aligned}
& \text { solution } 1 \\
& \qquad X_{+}=X_{+}(0) \exp \omega T, \\
& \quad X_{-}=X_{-}(0) \exp \omega T, \\
& \text { solution } \mathscr{2} \\
& \quad X_{+}=X_{+}(0) \cosh \omega\left(T-T_{0}\right), \\
& X_{-}=X_{-}(0) \sinh \omega\left(T-T_{0}\right), \\
& \text { solution } 3 \\
& \quad X_{+}=X_{+}(0) \sinh \omega\left(T-T_{0}\right), \\
& X_{-}=X_{-}(0) \cosh \omega\left(T-T_{0}\right),
\end{aligned}
$$

with $\omega=\sqrt{3 / 16}$. From these quantities, we construct $a(T)=\left(X_{-}\right)^{2 / 3}, b(T)=\left(X_{-} / X_{+}\right)^{1 / 3}$.

In Step 2, solutions is expressed with the original time variable $t$ via the temporal time $T$ as follows: 
solution 1

$$
\begin{aligned}
& t-t_{0}=\left(X_{-}(0) / X_{+}(0)\right)^{1 / 3} T, \\
& a(T)=\left(X_{-}(0)\right)^{2 / 3} \exp 2 \omega T / 3,
\end{aligned}
$$

solution 2

$$
\begin{aligned}
& t-t_{0}=\left(X_{-}(0) / X_{+}(0)\right)^{1 / 3} \int d T\left(\tanh \omega\left(T-T_{0}\right)\right)^{1 / 3} \\
& a(T)=\left(X_{-}(0) \sinh \omega\left(T-T_{0}\right)\right)^{2 / 3}
\end{aligned}
$$

solution 3

$$
\begin{aligned}
& t-t_{0}=\left(X_{-}(0) / X_{+}(0)\right)^{1 / 3} \int d T\left(\operatorname{coth} \omega\left(T-T_{0}\right)\right)^{1 / 3} \\
& a(T)=\left(X_{-}(0) \cosh \omega\left(T-T_{0}\right)\right)^{2 / 3}
\end{aligned}
$$

In the above solutions, $X_{ \pm}(0) \rightarrow$ (finite) $\times \exp \omega T_{0}$ with $T_{0} \rightarrow-\infty$ reproduce the previous solution $a(t)=a_{0} \sqrt{t-t_{0}}$ given in Eq.(18).

General solutions in these forms are more useful than the expression in Eq.(17). This is because $f(t)$ is not given in the explicite form in Eq. (17), and also because we must integrate further to obtain $a(t)$ from $f^{2}(t)=\frac{d a(t) / d t}{a(t)}$ even if we know the explicite form of $f$.

\section{Quantum solution in $R^{2}$ cosmology}

When we solve classical solution in pure $R^{2}$ gravity, we noticed that the auxiliary field formalism is quite powerful. Therefore, even in the quantum theory, we expect that it will become easy to solve the Wheeler-DeWitt equation when we use auxiliary field formalism.

Using the auxiliary field formalism, we start from the previous Lagrangian Eq.(22)

$$
\mathcal{L}=\hat{\alpha}\left\{\frac{16}{3 B} \dot{X}_{+} \dot{X}_{-}+B X_{+} X_{-}\right\}
$$

with $\hat{\alpha}=\alpha / 16 \pi G$. We define the canonical momentum in a standard way 


$$
\begin{aligned}
P_{+} & =\frac{\partial \mathcal{L}}{\partial \dot{X}_{+}}=\frac{16 \hat{\alpha}}{3 B} \dot{X}_{-}, \\
P_{-} & =\frac{\partial \mathcal{L}}{\partial \dot{X}_{-}}=\frac{16 \hat{\alpha}}{3 B} \dot{X}_{+}, \\
P_{B} & =\frac{\partial \mathcal{L}}{\partial \dot{B}} \approx 0 \quad \text { (primary constraint). }
\end{aligned}
$$

The Hamiltonian is given as follows:

$$
\mathcal{H}=P_{+} \dot{X}_{+}+P_{-} \dot{X_{-}}-\mathcal{L}=\frac{3 B}{16 \hat{\alpha}} P_{+} P_{-}-\hat{\alpha} B X_{+} X_{-}
$$

The secondary constraint, which comes from $\dot{P}_{B} \approx 0$, gives

$$
\dot{P}_{B}=\left\{\mathcal{H}, P_{b}\right\}_{\mathrm{cl} .}=\frac{3}{16 \hat{\alpha}} P_{+} P_{-}-\hat{\alpha} X_{+} X_{-} \approx 0
$$

Taking the Schrödinger representation $P_{+}=-i \frac{\partial}{\partial X_{+}}, \quad P_{-}=-i \frac{\partial}{\partial X_{-}}$, Eq.(35) gives the Wheeler-DeWitt equation of the form

$$
\left[\frac{\partial}{\partial X_{+}} \frac{\partial}{\partial X_{-}}+\frac{16 \hat{\alpha}^{2}}{3} X_{+} X_{-}\right] \Psi\left(X_{+}, X_{-}\right)=0 .
$$

In the above equation, the operator in square brackets is symmetry under the transformation

$$
\begin{aligned}
& X_{+} \rightarrow \ell X_{+}, \\
& X_{-} \rightarrow \frac{1}{\ell} X_{-},
\end{aligned}
$$

where $\ell$ is an arbitrary constant. If we find a solution $\Psi\left(X_{+}, X_{-}\right), \Psi\left(\ell X_{+}, \frac{1}{\ell} X_{-}\right)$also becomes a solution by using the transformation of Eq.(37) in Eq.(36). 
It is convenient to rewrite with variables $X_{0}, X_{1}$, which are defined by $X_{+}=\frac{1}{\ell}\left(X_{0}+X_{1}\right)$, $X_{-}=\ell\left(X_{0}-X_{1}\right)$. Using these variables, Eq.(36) becomes in the form

$$
\left[\left(\frac{\partial^{2}}{\partial X_{0}^{2}}-\frac{\partial^{2}}{\partial X_{1}^{2}}\right)+\frac{64 \hat{\alpha}^{2}}{3}\left(X_{0}^{2}-X_{1}^{2}\right)\right] \Psi\left(X_{+}, X_{-}\right)=0 .
$$

In order to find the eigenstate of the state vector, we put the state vector into the separable form $\Psi\left(X_{+}, X_{-}\right)=\Psi^{0}\left(X_{0}\right) \Psi^{1}\left(X_{1}\right)$. Using this separable form, the above equation becomes separated into two Wheeler-DeWitt equations, which are nothing but the Schrödinger equation of the harmonic oscillator with upside-down potential[23].

$$
\begin{aligned}
& \left(-\frac{\partial^{2}}{\partial X_{0}^{2}}-\frac{64 \hat{\alpha}^{2}}{3} X_{0}^{2}\right) \Psi^{0}\left(X_{0}\right)=E \Psi^{0}\left(X_{0}\right) \\
& \left(-\frac{\partial^{2}}{\partial X_{1}^{2}}-\frac{64 \hat{\alpha}^{2}}{3} X_{1}^{2}\right) \Psi^{1}\left(X_{1}\right)=E \Psi^{1}\left(X_{1}\right)
\end{aligned}
$$

Solutions are given by

$$
\Psi^{0}\left(X_{0}\right)=\Phi_{n}\left(X_{0}\right), \quad \Psi^{1}\left(X_{1}\right)=\Phi_{n}\left(X_{1}\right)
$$

with

$$
\begin{aligned}
& \Phi_{n}(X)=H_{n}\left(e^{-i \pi / 4} \beta X\right) e^{i \beta^{2} X^{2} / 2}, \\
& E=E_{n}=-2 i \beta^{2}(n+1 / 2), \quad \beta=\left(\frac{64 \hat{\alpha}^{2}}{3}\right)^{1 / 4}
\end{aligned}
$$

These $\Phi_{n}(X)$ are nothing but analytic continued forms from those of the harmonic oscillator. As equations are linear, superposition are also solutions. General solutions are given in the form

$$
\Psi\left(X_{+}, X_{-}\right)=\sum_{n=0}^{\infty}\left[c_{n} \Phi_{n}\left(X_{0}\right) \Phi_{n}\left(X_{1}\right)+d_{n} \Phi_{n}^{*}\left(X_{0}\right) \Phi_{n}^{*}\left(X_{1}\right)\right]
$$

Here we must comment on these solutions. First of all, these state vectors are not normalizable. The origin of this is because of the instability of the gravitational theory. According 
to the standard Copenhagen interpretation of the quantum theory, the above solutions are unphysical. But the above solutions are realy quite physical when we compare the above quantum solutions with classical solutions, as we will discuss on that later. Therefore, here in this paper, we take milder standpoint than the standard Copenhagen interpretation especially in quantum cosmology, and allow all solutions of the state vector without imposing the normalizability. As we do not impose the normalizability on the state vector, we obtain twice as many solutions compared to those of the harmonic oscillator.

\subsection{Correspondence between classical and quantum solutions un- der de Broglie-Bohm interpretation}

In this section, we study the connection between classical and quantum solutions to understand the problem of time in the general relativity. In the general relativity, because of the invariance under general coordinate transformation, the consequent Hamiltonian becomes weakly zero, then we come to the problem of time in canonical formalism. This is the problem because we may naively think that the weakly zero Hamiltonian will give only the solution of unrealistic static universe.

To overcome this unrealistic conclusion, many people take an approach from the WKB approximations [4, 6, 7]. The essential idea of the WKB approach is the followings. We first separate the Hamiltonian into classical and quantum parts, and we introduce time through the classical solution. Then the quantum Hamiltonian plays the role of the time development operator in the quantum system. Though classical and quantum Hamiltonians are both time developing, total Hamiltonian balanced to becomes zero. If there is matter field in the system, this approach may be reasonable, as the classical Hamiltonian may be dominated from the metric contribution and the quantum Hamiltonian may be dominated from the matter field. We cannot see the time dependence of the metric on the co-moving system, the matter may develop with time according to the quantum Hamiltonian.

We will not take this standard approach in our pure $R^{2}$ cosmology. Because there is no matter, it is unrealistic that classical and quantum contributions from the metric have the same magnitude. In the following, we take the de Broglie-Bohm interpretation 21 between classical and quantum solutions and try to study the problem of time.

In order to compare classical and quantum solutions in $R^{2}$ cosmology, we first consider the 
simplest state $\Psi_{0}$. The state vector in this case is given by $\Psi_{0}=e^{i \beta^{2}\left(X_{0}^{2}+X_{1}^{2}\right) / 2}=e^{i \beta^{2}\left(X_{+}^{2}+X_{-}^{2}\right) / 4}$. In general, we decompose the state vector into radial and phase components in the form $\Psi=A e^{i S}$. Then $S$ for $n=0$ state is given by $S=\frac{\beta^{2}}{4}\left(\ell^{2} X_{+}^{2}+\frac{1}{\ell^{2}} X_{-}^{2}\right)$.

According to the de Bloglie-Bohm interpretation, we obtain the first order differential equations for $X_{ \pm}$as

$$
\begin{aligned}
& \frac{16 \hat{\alpha}}{3} \dot{X}_{-}=P_{+}=\frac{\partial S}{\partial X_{+}}=\frac{\ell^{2} \beta^{2} X_{+}}{2}, \\
& \frac{16 \hat{\alpha}}{3} \dot{X}_{+}=P_{-}=\frac{\partial S}{\partial X_{-}}=\frac{\beta^{2} X_{-}}{2 \ell^{2}} .
\end{aligned}
$$

From the above equations and noticing relations $\beta^{2}=8 \hat{\alpha} / \sqrt{3}, \omega=\sqrt{3 / 16}$, we obtain $\dot{X}_{+}=$ $\ell^{-2} \omega X_{-}, \dot{X}_{-}=\ell^{2} \omega X_{+}$. Then we have the second order differential equation $\ddot{X}_{ \pm}=\omega^{2} X_{ \pm}$. The general solution is given by

$$
\begin{aligned}
& X_{+}=\frac{1}{\ell}\left(c_{1} e^{\omega T}+c_{2} e^{-\omega T}\right) \\
& X_{-}=\frac{\ell^{2} \dot{X}_{+}}{\omega}=\ell\left(c_{1} e^{\omega T}-c_{2} e^{-\omega T}\right) .
\end{aligned}
$$

If we compare these solutions with those of Eq.(25), the above solutions correspond to the classical solutions, Eqs.(26)-(28), exactly. The more detailed correspondence between the parameters in Eq.(44) and Eqs.(26)-(28) is given as follows with $\ell=\sqrt{X_{-}(0) / X_{+}(0)}$ :

$$
\begin{aligned}
& \text { solution } 1\left(c_{1}>0, c_{2}=0\right) \\
& c_{1}=\sqrt{X_{+}(0) X_{-}(0)} \\
& \text { solution } 2\left(c_{1}>0, c_{2}>0\right) \\
& c_{1}=\sqrt{X_{+}(0) X_{-}(0)} e^{-\omega T_{0}}, c_{2}=\sqrt{X_{+}(0) X_{-}(0)} e^{\omega T_{0}}, \\
& \text { solution } 3\left(c_{1}>0, c_{2}<0\right) \\
& c_{1}=\sqrt{X_{+}(0) X_{-}(0)} e^{-\omega T_{0}}, c_{2}=-\sqrt{X_{+}(0) X_{-}(0)} e^{\omega T_{0}} .
\end{aligned}
$$

Because of instability in $R^{2}$ cosmology, that is, the potential of the harmonic oscillator being upside-down, the solution becomes the sum of exponentially blowing up and damping 
term as time developes. This behavior of the classical solution is quite physical, we relax the standard Copenhagen interpretation and allow the unrenormalizable state vector in the quantum cosmology.

For general state vector $\Psi_{n}$ with $n \geq 2$, expressions obtained from the de Broglie-Bohm interpretation is different from expressions of classical solutions. This is because only $n=0$ and $n=1$ state vecotor is composed of only one term.

If the arguments of the Hermite polynomials become large, which describes that the universe becomes large, expressions constructed from the de Broglie-Bohm interpretation approach classical solutions. This is because the additional phase of wave functions takes constant value $-n \pi / 2$ in the above case, and constant phase does not contribute in $\partial S / \partial X_{ \pm}$.

According to de Broglie-Bohm interpretation, time comes through the relation $\dot{X}=P=\partial S / \partial X$, so that even if the Hamiltonian becomes zero system develops according to time.

In the rest of this section, we will point out the relation between de Broglie-Bohm interpretation and Ehrenfest theorem[5], which describes classical orbits as expectation values in quantum mechanics. For the one dimensional Hamiltonian $H=-\frac{1}{2 m} \frac{\partial^{2}}{\partial x^{2}}+V(x)$, the Ehrenfest theorem gives

$$
\frac{d<X>}{d t}=\frac{d}{d t} \int d x \psi^{*}(x, t) x \psi(x, t)=-\frac{i}{2 m} \int d x\left(\psi^{*} \frac{\partial \psi}{\partial x}-\frac{\partial \psi^{*}}{\partial x} \psi\right)
$$

after partial integration. While, according to the de Broglie-Bohm interpretation, we obtain

$$
m \frac{d X}{d t}=-\left.\frac{i}{2|\psi|^{2}}\left(\psi^{*} \frac{\partial \psi}{\partial x}-\frac{\partial \psi^{*}}{\partial x} \psi\right)\right|_{x=X}=\left.\frac{\partial S}{\partial x}\right|_{x=X} .
$$

If we compare Eq.(46) with Eq.(47), we can understand that de Borglie-Bohm interpretation is the local form of Ehrenfest theorem. Here we emphasize that the state vector is assumed to be normalizable in the proof of Ehrenfest theorem, while the normalizability of the state vector is not assumed in the local form of Eq.(47). In fact, the state vector is not normalizable in our examples. 


\section{Summary}

In this paper, we have studied various classical solutions in the generalized Einstein cosmology which contains higher derivative terms $R^{2}$. Especially we obtained general classical solutions in pure $R^{2}$ cosmology. Even in the quantum theory, we can solve the WheelerDeWitt equation in pure $R^{2}$ cosmology exactly. We compared classical and quantum solutions in this $R^{2}$ cosmology, and studied the problem of time using the de Broglie-Bohm interpretation. Further we pointed out the relation between de Broglie-Bohm interpretation and the Ehrenfest theorem.

Acknowledgement: Two of us (K.S. and K.U.) are grateful to the special research funds at Tezukayama University. 


\section{References}

[1] B. Hartle and S.W. Hawking, Phys. Rev. D28, 2960 (1983); S.W. Hawking, Nucl. Phys. B239, 257 (1984).

[2] A. Vilenkin, Phys. Rev. D32, 2511 (1985).

[3] A. Tomimatsu, "Quantum gravitational collapse of a scalar field and the wave function of black hole decay", Phys. Rev. D to appear.

[4] T. Banks, Nucl. Phys. B249, 332 (1985).

[5] J. Greensite, Nucl. Phys. B351, 749 (1989).

[6] S.P. de Alwis and D.A. MacIntire, Phys. Rev. D50, 5164 (1994).

[7] Y. Ohkuwa, "Semiclassical Time Variables in a Quantum Cosmology with a massless Scalar Field", MMMC-M-7 preprint.

[8] R. Utiyama and B.S. de Witt, J. Math. Phys. 3, 608 (1962).

[9] H. Nariai and K. Tomita, Progr. Theor. Phys. 46, 776 (1971).

[10] K. Stella, Gen. Rel. Grav. 9, 353 (1978); B. Whitt, Phys. Lett. 145B, 176 (1984); R. Utiyama, Progr. Theor. Phys. 72, 83 (1984).

[11] C. Brans and R.H. Dicke, Phys. Rev. 124, 925 (1961).

[12] Y. Fujii, Phys. Rev.D9, 874 (1974).

[13] R.H. Sanders, Astron. Astrophys. 136, L21 (1984).

[14] M. Kenmoku, E. Kitajima, Y. Okamoto and K. Shigemoto, Int. J. Mod. Phys. D2, 123 (1993).

[15] W-M. Suen and P.R. Anderson, Phys. Rev. D35, 2940 (1987).

[16] M.B. Mijić, M.S. Morris and W-M. Suen, Phys. Rev. D34, 2934 (1986).

[17] A.A. Starobinsky, Phys. Lett. 91B, 99 (1980).

[18] H. Schmidt, Phys. Rev. D49, 6354 (1994). 
[19] S. Weinberg, Gravitation and Cosmology (John Wiley and Sons, New York, 1972).

[20] M. Kenmoku, Y. Okamoto and K. Shigemoto, Phys. Rev.D48, 578 (1993).

[21] J.S. Bell, Speakable and Unspeakable in Quantum Mechanics (Cambridge Univ. Press, Cambridge, 1987).

[22] H. Suzuki, E. Takasugi and Y. Takayama, "Classically Integrable Cosmological Models with a Scalar Field", gr-qc/9508067 preprint.

[23] A.H. Guth and S-Y. Pi, Phys. Rev.D32, 1899 (1985). 